\title{
COMPARAÇÕES MÚLTIPLAS BAYESIANAS EM MODELOS NORMAIS HOMOCEDÁSTICOS E HETEROCEDÁSTICOS
}

\author{
Bayesian multiple comparisons in homocedastic and heterocedastic normal models
}

\author{
Paulo César de Resende Andrade1, Daniel Furtado Ferreira²
}

\begin{abstract}
RESUMO
Procedimentos de comparações múltiplas são utilizados para comparar médias de níveis de um fator, porém, os testes mais populares apresentam problemas de ambiguidade dos resultados e de controle do erro tipo I, além de terem seus desempenhos influenciados negativamente no caso de heterogeneidade de variâncias e não balanceamento. Objetivou-se, neste trabalho propor alternativas bayesianas para comparações múltiplas considerando os casos de homogeneidade e heterogeneidade de variâncias. A metodologia utilizada nesse trabalho foi baseada na distribuição a posteriori $t$ multivariada. Foram geradas $k$ cadeias de médias, utilizando o método de Monte Carlo. Foi obtida a amplitude padronizada sob $H_{0}$, obtida na distribuição a posteriori das médias, contemplando a possibilidade de se analisar tanto o caso de variâncias heterogêneas como o caso de variâncias homogêneas. Os procedimentos de comparações múltiplas bayesianos foram propostos com sucesso.
\end{abstract}

Termos para indexação: Simulação, erro tipo I, poder, Monte Carlo, não balanceamento.

\begin{abstract}
Multiple comparison procedures are used to compare factor's levels means, since the most popular tests show problems related to ambiguous results and to the control of the type I error rates. Moreover, their performance is worst in heterocedastics and unbalanced cases. The objective of this work is to propose a Bayesian alternative for multiple comparisons considering the homocedastic and heterocedastic normal models. The methodology adopted in this paper was based on a posteriori multivariate $t$ distribution. It was used k Monte Carlo chains of the mean factor to make inferences. The standardized range was obtained, under $H_{0}$, from the posteriori distribution of the means, for the analysis of homocedastic and heterocedastic cases. The bayesian procedures of multiple comparisons were successfully proposed.
\end{abstract}

Index terms: Simulation, type I error rate, power, Monte Carlo, unbalanced.

(Recebibo em 10 de abril de 2008 e aprovado em 23 de abril de 2010)

\section{INTRODUÇÃo}

$\mathrm{Na}$ experimentação, o pesquisador, geralmente, utiliza a análise de variância para avaliar a significância dos efeitos dos tratamentos, deparando-se com o problema de comparar médias de diferentes níveis do fator. Para verificar diferenças entre médias de tratamentos são sugeridos vários procedimentos de comparações múltiplas. $\mathrm{O}$ grande problema desses testes é a ambiguidade dos resultados (Machado et al., 2005). Um segundo problema, não menos importante, é o do controle do erro tipo I (Hochberg \& Tamhane, 1987; Hsu, 1996).

O problema da ambiguidade pode ser contornado com métodos alternativos de agrupamento, mas que apresentam a desvantagem de serem válidos apenas sob normalidade. Em situações de heterogeneidade de variâncias podem ser utilizados métodos de reamostragem bootstrap.

Uma alternativa é o uso de procedimentos bayesianos. Uma quantidade razoável de artigos, a maioria recente, leva em consideração o problema de comparações múltiplas do ponto de vista bayesiano. Duncan (1965) dá a primeira exposição completa de uma aproximação bayesiana para o problema de comparação múltipla. Waller \& Duncan (1969) ampliaram o procedimento original de Duncan (1965). Berry (1988) e Berry \& Hochberg (1999) usaram o processo Dirichlet como distribuição a priori no contexto de multiplicidades. Gopalan \& Berry (1998) desenvolveram essa aproximação, para obter probabilidades a posteriori para hipóteses de igualdade entre médias populacionais, com variâncias iguais.

Shaffer (1999) modificou o procedimento de Waller \& Duncan (1969), postulando prioris não informativas. Bratcher \& Hamilton (2005) apresentaram um procedimento de comparações múltiplas bayesiano para médias ranqueadas e obtidas de populações distribuídas normalmente. Cho et al. (2006) consideraram o problema específico de comparações múltiplas para populações com distribuição binomial negativa, aplicando a família Dirichlet,

${ }^{1}$ Universidade Federal dos Vales do Jequitinhonha e Mucuri/UFVJM - Instituto de Ciência e Tecnologia/ICT - Campus II - Rodovia MGT 367 - Km 583 - 5000 Alto da Jacuba - Diamantina, MG - paulo.andrade@ufvjm.edu.br

2Universidade Federal de Lavras/UFLA - Departamento de Ciências Exatas/DEX - Lavras, MG 
na forma de prioris. Porém, nenhum desses procedimentos enfoca o caso normal homocedástico ou heterocedástico.

Objetivou-se neste trabalho, propor alternativas bayesianas para comparações múltiplas considerando os casos de homogeneidade e heterogeneidade de variâncias em modelos com distribuição normal, balanceados ou não, ilustradas em exemplos simulados.

\section{MATERIAL E METODOS}

Considere o caso especial em que os elementos do vetor de observações $\boldsymbol{y}$ são amostras independentes $\boldsymbol{y}_{{ }_{1}}=$ $\left(y_{11}, \ldots, y_{1 n l}\right), \ldots, \boldsymbol{y}_{k}^{\prime}=\left(y_{k l}, \ldots, y_{k n K}\right)$, de tamanho $n_{l}, \ldots, n_{k}$ $\left(\Sigma_{n_{s}}=n\right)$ de $k$ populações com médias $\left(\theta_{l}, \ldots, \theta_{k}\right)$, respectivamente, e variância comum $\sigma^{2}$. Nesse caso, a distribuição a posteriori de $\theta$ é a $t$ multivariada, uma vez que foram usadas prioris conjugadas normal multivariada para o vetor de médias $\theta$ e Wishart invertida para a matriz de covariâncias. Assim, a distribuição a posteriori resultante é dada por

$p(\theta \mid y) \alpha\left[1+\frac{\sum_{i=1}^{k} n_{i}\left(\theta_{i}-\bar{y}_{i}\right)^{2}}{v s^{2}}\right]^{-\frac{v+k}{2}},-\propto<\theta_{i}<\propto, i=1, \ldots, k,(1)$

$\operatorname{com} \bar{y}_{i}=\frac{1}{n_{i}} \sum y_{i j}, v=n-k, e s^{2}=\frac{1}{n-k} \sum_{i} \sum_{j}\left(y_{i j}-\bar{y}_{i}\right)^{2}$ que foi usada para se realizar as comparações de médias de $k$ populações normais. Essa distribuição a posteriori é a distribuição $t$ multivariada de dimensão $k \operatorname{com} n$ graus de liberdade, centrada na média amostral $\bar{y}_{i}$, conforme detalhamento apresentado na sequência.

A idéia é, a partir da $t$ multivariada, propor um procedimento bayesiano para se realizar as comparações múltiplas das médias.

\section{Geração de uma amostra de tamanhon da t multivariada}

Seja $k$ populações com médias $\theta=\left(\theta_{1}, \theta_{2}, \ldots, \theta_{k}\right)^{t}$. Observações $\boldsymbol{Y}=\left\{Y_{1}, Y_{2}, \ldots, Y_{k}\right\}$ são obtidas dessas populações, em que $Y_{i}=\left(\boldsymbol{y}_{\boldsymbol{i} \boldsymbol{1}}, \boldsymbol{y}_{\boldsymbol{i}}, \ldots, \boldsymbol{y}_{\boldsymbol{i \boldsymbol { n } _ { \boldsymbol { i } }}}\right)^{t}$ é um vetor de observações $n_{i} \times 1$ condicionalmente independentes no tratamento $i, i=1,2, \ldots, k$ e $\sum_{i=1}^{k} n_{i}=n$. O problema de comparações múltiplas (PCM) é fazer inferências sobre as relações entre os $\theta$ baseados em $\boldsymbol{Y}=\left\{Y_{1}, Y_{2}, \ldots, Y_{k}\right\}$.

Pela equação (1) pode-se observar que a distribuição a posteriori do parâmetro $\theta$ foi utilizada para se realizar comparações múltiplas de médias normais. Considerando o modelo na forma $y_{i j}=\mu+\tau_{i}+{ }_{i j} \mathbb{e}$ faz-se $\theta=\mu_{i}=\mu+$ $\tau_{i}$. A distribuição $t$ multivariada, $t(n, \mu, \Sigma v)$, possui parâmetros que podem ser especificados por:

$\mu=\bar{y}=\left[\begin{array}{c}\bar{y}_{1} \\ \bar{y}_{2} \\ \vdots \\ \bar{y}_{k}\end{array}\right], \Sigma=S_{\bar{y}}=\left[\begin{array}{cccc}\frac{s_{1}{ }^{2}}{n_{1}} & 0 & \cdots & 0 \\ 0 & \frac{s_{2}{ }^{2}}{n_{2}} & \cdots & 0 \\ \vdots & \vdots & \ddots & \vdots \\ 0 & 0 & \cdots & \frac{s_{k}{ }^{2}}{n_{k}}\end{array}\right], v=n-k$

em que $n=\sum_{i=1}^{k} n_{i}$ é o número de médias populacionais e $\Sigma$ é a matriz de covariâncias das médias.

Os elementos localizados "fora" da diagonal principal indicaram covariâncias nulas entre as populações, sendo considerados independentes, ao passo que o $k$ ésimo elemento da diagonal de $\Sigma$ representa a variância da $k$-ésima população sobre o número de elementos da mesma.

Na sequência foi considerada a forma geral usada para gerar variáveis aleatórias $k$-dimensionais $t$ multivariada com $n$ graus de liberdade e parâmetros $\mu$ e $\Sigma$. Seja um vetor aleatório $\boldsymbol{X}$ com distribuição $N_{k}(\boldsymbol{0}, \Sigma)$ e a variável escalar $\boldsymbol{U}$ com distribuição qui-quadrado com $n$ graus de liberdade, então o vetor aleatório $\boldsymbol{Y}$ dado pela transformação $Y=\sqrt{v} \frac{X}{\sqrt{U}}+\mu$, possui distribuição $t$ multivariada com $v$ graus de liberdade.

Foi aplicada a transformação anterior $n$ vezes, a $n$ diferentes vetores aleatórios $\boldsymbol{X}$ e a $n$ variáveis escalares $\boldsymbol{U}$, tendo ao final do processo uma amostra de tamanho $n$ da distribuição $t$ multivariada com $n$ graus de liberdade, utilizando o programa R (R Development Core Team, 2006), como ambiente de programação, em que todos os procedimentos foram implementados.

\section{Distribuição nula da amplitude padronizada}

A partir da distribuição a posteriori $t$ multivariada, $t(n, \mu, \Sigma, n)$, foram geradas $k$ cadeias de médias $\mu_{i}$, utilizando o método de Monte Carlo e assumindo médias constantes, vetor $\mu=(\mu, \mu, \ldots, \mu)^{\mathrm{t}}$, ou seja, todos os componentes iguais. Assim, sem perda de generalidade, foi assumido $\mu=0$ (para todos os $k$ componentes), impondo a hipótese nula $H_{0}$ no método bayesiano.

No próximo passo foi realizada a geração da amplitude padronizada da posteriori, sob $H_{0}$, e obtida na distribuição a posteriori das médias da seguinte forma: 


$$
q=\frac{\operatorname{máx}\left(\mu_{i}\right)-\min \left(\mu_{i}\right)}{\sigma_{h}}
$$

em que $\sigma_{h}$ representa a média harmônica das variâncias das $k$ médias, dada por:

$$
\sigma_{h}=\sqrt{\frac{1}{\frac{1}{k}\left(\frac{n_{1}}{s_{1}{ }^{2}}+\frac{n_{2}}{s_{2}{ }^{2}}+\cdots+\frac{n_{k}}{s_{k}{ }^{2}}\right)}}
$$

para contemplar a possibilidade de se analisar tanto o caso de variâncias heterogêneas como o caso de variâncias homogêneas.

O fato de se gerar a amplitude padronizada da posteriori, sob $H_{0}$, obtida da distribuição a posteriori das médias da forma mostrada na equação (3) tem um significado muito importante, pois possibilita a análise sob heterogeneidade de variâncias e sob condições de não balanceamento, diferentemente dos testes de comparações múltiplas convencionais.

Calcula-se a seguir a diferença mínima significativa $\Delta=\sigma_{h} \cdot \theta_{a .}$, em que $\theta_{a .}$ é o quantil superior $100 \alpha \%$ da distribuição a posteriori de $q$. Para qualquer amplitude maior que $\Delta$, a diferença é significativamente considerada diferente de zero.

Para descrever os resultados finais das análises do método proposto neste trabalho foram considerados quatro exemplos simulados, sob duas situações. $\mathrm{Na}$ primeira situação, foram simulados dados de experimentos sob $H_{0}$. Na segunda, foram simulados dados de experimentos sob $H_{1}$, sendo que a diferença entre as médias dos níveis do fator foi de dois erros padrões. Em ambos foram consideradas situações balanceadas e homocedásticas e não balanceadas e heterocedásticas.

Foram obtidas as densidades a posteriori da distribuição da amplitude padronizada $q$ e os quantis superiores $10 \%, 5 \%$ e $1 \%$ dessa distribuição, em cada um dos quatro exemplos utilizados, utilizando-se um estimador de densidades Kernel do programa R (R Development Core Team, 2006).

Para realizar a inferência a respeito da hipótese $H_{0}$ : $\mu_{i}=\mu_{i}, i \neq i=1,2, \ldots, k$, considerando todos os pares e todos os exemplos, foi obtido

$$
\Delta=\sigma_{h} \cdot q_{\alpha},
$$

para $a=10 \%, 5 \%$ e $1 \%$ sendo $\sigma_{h}$ obtido a partir da expressão(3).
Da mesma forma foi obtida a média na distribuição a posteriori de $\mu$ e assim, para cada $\operatorname{par}\left(\mu_{i}, \mu_{i}\right)$, foi obtido o intervalo de confiança bayesiano

$\operatorname{ICB}_{1-\alpha}\left(\mu_{i}-\mu_{i^{\prime}}\right): \bar{\mu}_{i}-\bar{\mu}_{i^{\prime}} \pm \Delta$

em que $\bar{\mu}_{i}$ e $\bar{\mu}_{i}$ são as médias posteriori das cadeias de $\mu_{i}$ e $\mu_{i}$. Todos os intervalos que contiverem zero indicarão que a hipótese $H_{0}: \mu_{i}=\mu_{i}$, não deve ser rejeitada; se os limites do $\mathrm{ICB}_{1-\mathrm{a}}$ forem positivos, indicarão que $\mu_{i}>\mu_{i}$, ; se forem negativos, $\mu_{i}<\mu_{i}$,

Duas alternativas foram avaliadas. Na primeira, considerando todos os pares $\left(\mu_{i}, \mu_{i}\right)$, foi calculada a seguinte probabilidade a posteriori

$\mathrm{P}\left(\mu_{i}>\mu_{i}\right)$.

Esta probabilidade serviu de forma descritiva como uma evidência a favor ou contra $H_{0}$.

A segunda alternativa seria obter uma nova cadeia com os limites inferior $\left(L I^{i{ }^{\prime}}\right)$ e superior $\left(L S^{i i^{\prime}}\right)$ de um intervalo a posteriori para cada par de médias, da seguinte forma

$\left\{\begin{array}{l}L I^{i i^{\prime}}=\mu_{i j}-\mu_{i^{\prime} j}-q_{j} \sigma_{h} \\ L S^{i i^{\prime}}=\mu_{i j}-\mu_{i^{\prime} j}+q_{j} \sigma_{h}\end{array}\right.$

Como medida de evidência a favor ou contra $H_{0}$ : $\mu_{i}=\mu_{i}$, calculou-se a probabilidade $a$ posteriori dos intervalos conterem o valor zero - $P(I C B \supset 0)$. Seja $I(0$ $\left.\in\left[L I_{j}{ }^{i{ }^{\prime}}, L S_{j}{ }^{i{ }^{\prime}}\right]\right)$, a função indicadora para verificar se o valor $O$ pertence ao intervalo na j-ésima unidade amostral de Monte Carlo da cadeia a posteriori, logo,

$P(I C B \supset 0)=\frac{{ }_{j=1}^{n} \boldsymbol{I}\left(\boldsymbol{O}\left[\boldsymbol{L I}{ }_{j}{ }^{i^{\prime}}, \boldsymbol{L} \boldsymbol{S}_{j}{ }^{\left.{ }^{i{ }^{\prime}}\right]}\right]\right)}{n}$

Uma última análise foi realizada utilizando o HPD nas cadeias a posteriori da distribuição das diferenças de duas médias. Para cada cadeia $\mu_{i}-\mu_{i}$, foi obtido o HPD utilizando um coeficiente de credibilidade de $95 \%$, utilizando se para isso o pacote BOA, um aplicativo do programa $\mathrm{R}$ (R Development Core Team, 2006). Os resultados de todos os procedimentos foram comparados em experimentos simulados.

Foram simulados dois exemplos sob a hipótese nula, ou seja, todas as médias dos níveis do fator foram consideradas iguais. No primeiro caso, considerou-se uma 
situação balanceada e homocedástica. Foi considerado o valor $k=6$ e $r=5$, com $\sigma^{2}=1$. Assim, as observações $y_{i j}$ foram geradas de uma normal $\mathrm{N}(0,1)$, para $\mathrm{i}=1,2, \ldots, k \mathrm{e} \mathrm{j}=$ $1,2, \ldots, r$.

No segundo caso, considerou-se que as amostras de cada população seriam geradas de distribuições normais com média 0 e variâncias $\sigma_{1}{ }^{2}=1, \sigma_{2}{ }^{2}=4, \sigma_{3}{ }^{2}=7, \sigma_{4}{ }^{2}=$ $10, \sigma_{5}{ }^{2}=13$ e $\sigma_{6}^{2}=16$. Isso foi realizado para que houvesse uma heterogeneidade de variâncias máxima de $\delta=\sigma_{6}{ }^{2} / \sigma_{1}{ }^{2}=16$. Assim, os valores $y_{i j}$ foram gerados de $\mathrm{N}\left(0, \sigma_{\mathrm{i}}{ }^{2}\right)$, considerando os valores das médias dos $n_{\mathrm{i}}$ 's dados por 3, 5, 4, 3, 2 e 4, para $\mathrm{i}=1,2,3,4,5$ e 6 , respectivamente.

Foram simulados dois exemplos sob a hipótese alternativa, sendo o primeiro balanceado e homocedástico e o segundo não balanceado e heterocedástico. Os valores de $k, r$ ou $n_{\mathrm{i}}$ e $\sigma^{2}$ ou $\sigma_{\mathrm{i}}{ }^{2}$ foram os mesmos. A diferença é que as amostras das populações consideradas possuíam diferentes médias. A média da população 1 foi considerada igual a zero $\left(\mu_{i}=0\right)$ sem perda de generalidade. As demais médias $\mu_{i}$ foram definidas por:

$$
\mu_{\mathrm{i}+1}=\mu_{\mathrm{i}}+2 \sigma_{\mathrm{h}}
$$

sendo $\sigma_{\mathrm{h}}$ a raiz quadrada da média harmônica das variâncias das médias $\mu_{\mathrm{i}}$, dada por:

$$
\sigma_{h}=\sqrt{\frac{1}{\frac{1}{k}\left(\frac{n_{1}}{\sigma_{1}^{2}}+\frac{n_{2}}{\sigma_{2}^{2}}+\cdots+\frac{n_{k}}{\sigma_{k}^{2}}\right)}}
$$

\section{RESULTADOS E DISCUSSÃO}

Foram considerados $H_{0}$ e $H_{1}$ em dois casos, experimentos balanceados e homocedásticos (comportados) e não balanceados e heterocedásticos (não comportados).

A discussão é prejudicada pela escassez de artigos sobre o problema.

As médias originais e a posteriori de experimentos simulados sob $H_{0}$ e $H_{1}$, comportado e não comportado apresentaram resultados similares, indicando que a simulação foi consistente. É conveniente salientar que cada média simulada difere da outra por uma diferença de dois erros padrões, sob $H_{1}$. Assim, as diferenças podem ser atribuídas apenas ao erro de Monte Carlo, obtido nas 10.000 simulações realizadas.

As densidades a posteriori permitem que se afirme que houve uma grande flexibilidade do método, indicado pelo ajuste ao conjunto de dados e as suas características. Assim, diferentemente do caso clássico, os quantis superiores da amplitude padronizada modificam-se em função do não balanceamento e da heterogeneidade de variâncias que porventura possam existir nos conjuntos de dados submetidos à análise.

$\mathrm{Na}$ Tabela 1 são apresentados os quantis superiores $10 \%, 5 \%$ e $1 \%$ da distribuição da amplitude padronizada $q$. Os valores dos quantis da amplitude padronizada são muito similares aos da amplitude estudentizada $q$ do teste clássico de comparações múltiplas de Tukey para médias de 6 fatores com 24 graus de liberdade, cujos valores são $3,90,4,37$ e 5,37 para $10 \%, 5 \%$ e $1 \%$, respectivamente, principalmente com os do caso comportado. Os valores observados sob $H_{1}$ comportado e não comportado indicam uma pequena diferença, sendo um pouco maiores nesse último caso.

Considerando as diferenças entre todos os pares de médias e comparando com o $\Delta$, obtido a partir da equação (4), verifica-se que realmente não há diferença significativa entre as médias dos fatores nas situações sob $H_{0}$ comportado e não comportado. Esse resultado é muito interessante, pois ambas as situações foram simuladas sob a hipótese nula em que as médias dos fatores são iguais e com isso não se esperava detectar diferenças entre as médias. Verifica-se também que há diferenças significativas entre as médias dos fatores nas situações sob $H_{1}$ comportado e não comportado, mas que muitas diferenças foram não significativas, indicando que se cometeu o erro tipo II. A heterogeneidade de variâncias afetou consideravelmente o poder do teste. As diferenças mínimas significativas foram muito discrepantes, sendo uma delas mais de

Tabela 1 - Quantis superiores da amplitude padronizada q da distribuição a posteriori.

\begin{tabular}{ccccc}
\hline \multirow{2}{*}{$1-\alpha$} & \multicolumn{2}{c}{ Sob H $_{0}$} & \multicolumn{2}{c}{ Sob H $_{1}$} \\
\cline { 2 - 5 } & Comportado & Não Comportado & Comportado & Não Comportado \\
\hline $90 \%$ & 3,877603 & 4,278497 & 3,933255 & 4,286779 \\
$95 \%$ & 4,373322 & 4,849312 & 4,421675 & 4,873247 \\
$99 \%$ & 5,389085 & 6,114813 & 5,374668 & 6,203168 \\
\hline
\end{tabular}

Ciênc. agrotec., Lavras, v. 34, n. 4, p. 845-852, jul./ago., 2010 
quatro vezes superior à outra. Isso é um indicativo que o teste quando aplicado em situações heterocedásticas, deve ser menos poderoso.

Observando os intervalos de confiança bayesianos dos testes de comparações múltiplas, utilizando os quantis $95 \%$ da distribuição da amplitude padronizada $a$ posteriori $q$ sob $H_{0}$ comportado e não comportado, verifica-se que todos os intervalos englobaram o zero, que é um indicativo de que a hipótese $H_{0}: \mu_{i}=\mu_{i}$, não deva ser rejeitada. Esse procedimento é alternativo ao procedimento anterior, considerando a amplitude padronizada a posteriori. Outro fato interessante a ser destacado é que os intervalos de confiança bayesianos para a situação não comportada são mais amplos. Isso é decorrente da maior diferença mínima bayesiana nesse caso, em consequência da maior variabilidade no caso heterocedástico.

Assim, o procedimento é adequado tanto para situações comportadas como para situações em que as condições não seriam favoráveis à aplicação de um teste clássico de comparações múltiplas. Em ambos os casos, no entanto, a suposição de normalidade não foi violada. Sob $H_{1}$ comportado e não comportado, intervalos contiveram o valor zero, que é um indicativo de que está se cometendo o erro tipo II. Cada média populacional, nesse caso, difere da sua vizinha por dois erros padrões.
Médias vizinhas de um salto igual a 2, possuem valores distantes em quatro erros padrões e assim sucessivamente. Assim, médias mais distantes tendem a ser diferenciadas. As oscilações nos valores das médias experimentais são devidas aos maiores erros associados à situação denominada não comportada.

As probabilidades a posteriori $\mathrm{P}\left(\mu_{\mathrm{i}}>\mu_{\mathrm{i}}\right)$, considerando todos os pares $\left(\mu_{\mathrm{i}}, \mu_{\mathrm{i}}\right)$ da distribuição conjunta a posteriori, são apresentadas na Tabela 2 . Quando a probabilidade $\mathrm{P}\left(\mu_{\mathrm{i}}>\mu_{\mathrm{i}}\right) \leq 0,025 \mathrm{ou}$ $\mathrm{P}\left(\mu_{\mathrm{i}}>\mu_{\mathrm{i}}\right) \geq 0,975$ tem-se uma evidência contra $H_{0}$, caso $0,025<\mathrm{P}\left(\mu_{\mathrm{i}}>\mu_{\mathrm{i}}\right)<0,975$, tem-se uma evidência a favor de $H_{0}$. Essa evidência foi considerada neste estudo por meio da escolha de $95 \%$ de confiança, para termos uma situação comparável ao normalmente aplicado aos testes clássicos de comparações múltiplas. Verifica-se que, sob $H_{0}$ comportado, todas as probabilidades evidenciam que a hipótese $H_{0}$ não deve ser rejeitada. Contudo, sob $H_{0}$ não comportado, há uma situação de evidência contra $H_{0}$, parâmetros $\mu_{4}$ e $\mu_{6}$, que indicam que o procedimento é mais liberal, com mais chance de cometer erros. Verifica-se também que, sob $H_{1}$ comportado, há 4 (quatro) casos evidenciando que a hipótese $H_{0}$ não deve ser rejeitada, e que, sob $H_{1}$ não comportado, há 5 (cinco) casos. Esses resultados, quando comparados com aqueles que utilizaram

Tabela 2 - Probabilidades a posteriori $\mathrm{P}\left(\mu_{\mathrm{i}}>\mu_{\mathrm{i}}\right)$, em porcentagem, considerando todos os pares $\left(\mu_{\mathrm{i}}, \mu_{\mathrm{i}},\right)$ distribuição conjunta a posteriori.

\begin{tabular}{ccccc}
\hline \multirow{2}{*}{ Parâmetros } & \multicolumn{2}{c}{ Sob $\mathrm{H}_{0}$} & \multicolumn{2}{c}{ Sob $\mathrm{H}_{1}$} \\
\cline { 2 - 4 } & Comportado & Não Comportado & Comportado & Não Comportado \\
\hline$\mu_{1}$ e $\mu_{2}$ & 68,77 & 58,56 & 22,29 & 32,50 \\
$\mu_{1}$ e $\mu_{3}$ & 80,41 & 75,15 & 6,41 & 0,03 \\
$\mu_{1}$ e $\mu_{4}$ & 40,65 & 92,84 & 0,00 & 15,73 \\
$\mu_{1}$ e $\mu_{5}$ & 55,21 & 37,54 & 0,00 & 0,01 \\
$\mu_{1}$ e $\mu_{6}$ & 74,08 & 21,90 & 0,00 & 2,35 \\
$\mu_{2}$ e $\mu_{3}$ & 65,21 & 70,24 & 21,88 & 0,00 \\
$\mu_{2}$ e $\mu_{4}$ & 23,79 & 92,23 & 0,06 & 24,12 \\
$\mu_{2}$ e $\mu_{5}$ & 36,12 & 29,55 & 0,00 & 0,00 \\
$\mu_{2}$ e $\mu_{6}$ & 56,75 & 13,52 & 0,00 & 0,86 \\
$\mu_{3}$ e $\mu_{4}$ & 13,74 & 82,42 & 0,53 & 76,70 \\
$\mu_{3}$ e $\mu_{5}$ & 23,38 & 17,95 & 0,00 & 0,12 \\
$\mu_{3}$ e $\mu_{6}$ & 41,31 & 6,35 & 0,00 & 99,29 \\
$\mu_{4}$ e $\mu_{5}$ & 64,12 & 5,92 & 1,89 & 26,51 \\
$\mu_{4}$ e $\mu_{6}$ & 81,04 & 1,45 & 0,13 & 0,08 \\
$\mu_{5}$ e $\mu_{6}$ & 69,74 & 35,79 & 8,76 & \\
\hline
\end{tabular}


a distribuição a posteriori da amplitude padronizada permitem que se infira que este método é mais poderoso do que o anterior. No entanto, sob a hipótese nula, principalmente sob situações não comportadas, esse procedimento foi considerado mais liberal. Assim, a vantagem em relação ao maior poder deve ser olhada com ressalva. Esse procedimento é o que os bayesianos normalmente fazem, ou seja, tomam suas decisões baseadas na distribuição a posteriori conjunta dos parâmetros e numa medida probabilística a favor de uma hipótese ou de outra.

Assim, este procedimento, aplicado para fins de comparação, não é equivalente ao da proposta de trabalho que considera a distribuição a posteriori da amplitude padronizada para construir o critério de decisão. $O$ teste de Tukey seria a alternativa equivalente ao procedimento bayesiano que considera a amplitude a posteriori padronizada e que controlaria as taxas de erro por experimento. Portanto, a maior liberalidade dessa alternativa seria esperada, como acontece com os seus pares clássicos.

As probabilidades a posteriori dos intervalos de confiança conter o valor zero - $P(I C B \supset 0)$, são apresentadas na Tabela 3. A diferença entre esse método e o anterior é que as cadeias das diferenças de médias consideram os valores de $\Delta$ determinados em cada ponto amostral dessa cadeia. Assim, espera-se uma maior proteção do erro tipo I quando se utiliza tal procedimento.
Quando a probabilidade for menor ou igual a 5\% tem-se uma evidência contra $\mathrm{H}_{0}$, caso contrário, a favor de $H_{0}$.

Verifica-se que, sob $H_{0}$, todas as probabilidades constituíram-se em uma forte evidência a favor de $H_{0}$. Esse procedimento é equivalente ao intervalo de confiança bayesiano usando o $\Delta$ e a diferença entre eles é que o intervalo bayesiano é obtido como um sumário da cadeia da diferença entre duas médias e a obtenção das probabilidades, envolve o cálculo do intervalo em cada ponto simulado da cadeia a posteriori. Verifica-se também que, sob $H_{1}$ existem probabilidades que evidenciam uma decisão a favor de $H_{0}$, ou seja, indicativo de se estar cometendo erro do tipo II. Esse procedimento apresentou piores resultados do que os obtidos utilizando o intervalo baseado na média a posteriori das cadeias da diferença das médias e com a amplitude padronizada, pois houve mais erros do tipo II no presente caso.

Os HPD's de $95 \%$ para cada cadeia $\mu_{i}=\mu_{i}$, são apresentados na Tabela 4. Novamente é interessante que se saliente que esse é um procedimento que seria genuinamente adotado pelos estatísticos bayesianos.

Esse procedimento deve ser melhor do que os procedimentos que utilizam a distribuição a posteriori das diferenças de médias apresentados anteriormente, mas não contorna o problema do controle do erro tipo I global nas diferentes análises realizadas simultaneamente.

Tabela 3 - Probabilidades, em porcentagem, do intervalo de confiança bayesiano (56) a posteriori conter o valor zero.

\begin{tabular}{ccccc}
\hline \multirow{2}{*}{ Parâmetros } & \multicolumn{2}{c}{ Sob $\mathrm{H}_{0}$} & \multicolumn{2}{c}{ Sob $\mathrm{H}_{1}$} \\
\cline { 2 - 5 } & Comportado & Não Comportado & Comportado & Não Comportado \\
\hline$\mu_{1} \mathrm{e} \mu_{2}$ & 84,28 & 87,92 & 78,90 & 86,29 \\
$\mu_{1} \mathrm{e} \mu_{3}$ & 77,63 & 81,19 & 58,13 & 44,61 \\
$\mu_{1} \mathrm{e} \mu_{4}$ & 86,40 & 57,03 & 2,89 & 2,82 \\
$\mu_{1} \mathrm{e} \mu_{5}$ & 86,74 & 80,27 & 0,02 & 67,23 \\
$\mu_{1} \mathrm{e} \mu_{6}$ & 81,35 & 78,52 & 0,00 & 0,88 \\
$\mu_{2} \mathrm{e} \mu_{3}$ & 85,61 & 86,83 & 79,24 & 59,60 \\
$\mu_{2} \mathrm{e} \mu_{4}$ & 79,95 & 63,98 & 8,74 & 4,33 \\
$\mu_{2} \mathrm{e} \mu_{5}$ & 85,43 & 80,44 & 0,16 & 78,00 \\
$\mu_{2} \mathrm{e} \mu_{6}$ & 86,53 & 76,48 & 0,01 & 1,34 \\
$\mu_{3} \mathrm{e} \mu_{4}$ & 71,73 & 76,06 & 22,49 & 28,68 \\
$\mu_{3} \mathrm{e} \mu_{5}$ & 79,28 & 70,60 & 0,92 & 76,40 \\
$\mu_{3} \mathrm{e} \mu_{6}$ & 86,39 & 62,41 & 0,10 & 13,74 \\
$\mu_{4} \mathrm{e} \mu_{5}$ & 85,26 & 45,61 & 38,68 & 14,62 \\
$\mu_{4} \mathrm{e} \mu_{6}$ & 76,87 & 33,07 & 8,77 & 82,51 \\
$\mu_{5} \mathrm{e} \mu_{6}$ & 83,76 & 80,92 & 64,70 & 6,85 \\
\hline
\end{tabular}


Tabela 4 - HPD's de 95\% para cada cadeia $\mu_{\mathrm{i}}-\mu_{\mathrm{i}}$.

\begin{tabular}{|c|c|c|c|c|c|c|c|c|}
\hline \multirow{3}{*}{ Parâmetros } & \multicolumn{4}{|c|}{ Sob $\mathrm{H}_{0}$} & \multicolumn{4}{|c|}{ Sob $\mathrm{H}_{1}$} \\
\hline & \multicolumn{2}{|c|}{ Comportado } & \multicolumn{2}{|c|}{ Não Comportado } & \multicolumn{2}{|c|}{ Comportado } & \multicolumn{2}{|c|}{ Não Comportado } \\
\hline & LIC & LSC & LIC & LSC & LIC & LSC & LIC & LSC \\
\hline$\mu_{1}$ e $\mu_{2}$ & $-0,81$ & 1,341 & $-2,78$ & 3,213 & $-1,36$ & 0,620 & $-4,56$ & 2,917 \\
\hline$\mu_{1}$ e $\mu_{3}$ & $-0,57$ & 1,552 & $-1,95$ & 4,092 & $-1,72$ & 0,222 & $-7,67$ & 0,117 \\
\hline$\mu_{1}$ e $\mu_{4}$ & $-1,16$ & 0,978 & $-0,77$ & 5,801 & $-3,01$ & $-1,05$ & $-12,7$ & $-4,46$ \\
\hline$\mu_{1}$ e $\mu_{5}$ & $-0,97$ & 1,160 & $-4,39$ & 3,002 & $-4,08$ & $-2,12$ & $-7,10$ & 2,158 \\
\hline$\mu_{1}$ e $\mu_{6}$ & $-0,77$ & 1,387 & $-4,17$ & 1,954 & $-4,75$ & $-2,80$ & $-13,8$ & $-6,05$ \\
\hline$\mu_{2}$ e $\mu_{3}$ & $-0,87$ & 1,249 & $-1,92$ & 3,497 & $-1,36$ & 0,581 & $-6,54$ & 0,330 \\
\hline$\mu_{2}$ e $\mu_{4}$ & $-1,43$ & 0,673 & $-0,86$ & 5,139 & $-2,63$ & $-0,65$ & $-11,5$ & $-4,01$ \\
\hline$\mu_{2}$ e $\mu_{5}$ & $-1,26$ & 0,889 & $-4,19$ & 2,559 & $-3,66$ & $-1,73$ & $-5,72$ & 2,929 \\
\hline$\mu_{2}$ e $\mu_{6}$ & $-0,92$ & 1,222 & $-4,24$ & 1,176 & $-4,39$ & $-2,42$ & $-12,5$ & $-5,64$ \\
\hline$\mu_{3}$ e $\mu_{4}$ & $-1,63$ & 0,506 & $-1,75$ & 4,415 & $-2,33$ & $-0,37$ & $-8,67$ & $-0,81$ \\
\hline$\mu_{3}$ e $\mu_{5}$ & $-1,41$ & 0,735 & $-5,02$ & 1,980 & $-3,35$ & $-1,38$ & $-2,69$ & 6,120 \\
\hline$\mu_{3}$ e $\mu_{6}$ & $-1,20$ & 0,934 & $-5,17$ & 0,627 & $-3,96$ & $-1,99$ & $-9,70$ & $-2,51$ \\
\hline$\mu_{4}$ e $\mu_{5}$ & $-0,89$ & 1,248 & $-6,86$ & 0,627 & $-2,02$ & $-0,09$ & 1,723 & 11,13 \\
\hline$\mu_{4}$ e $\mu_{6}$ & $-0,54$ & 1,588 & $-6,65$ & $-0,39$ & $-2,70$ & $-0,74$ & $-5,11$ & 2,546 \\
\hline$\mu_{5}$ e $\mu_{6}$ & $-0,80$ & 1,334 & $-4,16$ & 2,916 & $-1,62$ & 0,298 & $-11,8$ & $-3,09$ \\
\hline
\end{tabular}

Verifica-se que esse procedimento foi equivalente ao das probabilidades a posteriori $\mathrm{P}\left(\mu_{\mathrm{i}}>\mu_{\mathrm{i}}\right)$, nesse caso. Espera-se que resultados similares entre esses dois procedimentos sejam observados com muita frequência em situações práticas reais, mas não há garantias de equivalência teórica entre esses dois métodos. O que, no entanto, fica claro é que ambos os métodos não possuem garantias de controle do erro tipo I simultaneamente nas múltiplas inferências realizadas nas cadeias a posteriori das diferenças entre médias. Assim, observando-se o erro tipo I no caso não comportado, tem-se uma evidência de que esse procedimento seja mais liberal que os que utilizam a distribuição a posteriori da amplitude padronizada.

\section{CONCLUSÕES}

$\mathrm{O}$ procedimento que encontra os intervalos de confiança $\mathrm{ICB}_{1-\alpha}\left(\mu_{i}-\mu_{i}\right)$ usando a amplitude padronizada $\Delta$ é mais conservativo e flexível a situações de heterocedasticidade. Apresenta resultados praticamente equivalentes ao método que calcula as probabilidades do intervalo a posteriori conter o zero - $P(I C B \supset 0)$.

$\mathrm{O}$ procedimento que calcula as probabilidades $a$ posteriori $\mathrm{P}\left(\mu_{\mathrm{i}}>\mu_{\mathrm{i}}\right)$ e os HPD's, foram equivalentes entre si. São também mais liberais, identificando diferenças em situações em que elas não existem.
Os procedimentos propostos apresentam a desvantagem de ainda não estarem implementados. Em compensação apresentam vantagens em relação aos testes convencionais, no sentido de não haver necessidade de balanceamento dos dados, que é muito significativo do ponto de vista prático, e de poderem ser utilizados em situações homo e heterocedásticas.

Os procedimentos baseados na amplitude padronizada foram superiores aos demais procedimentos estudados por terem controlado, nos exemplos simulados, o erro tipo I e detectadas a maior parte das diferenças sob $H_{l}$.

Os procedimentos de comparações múltiplas bayesianos foram propostos com sucesso para situações de normalidade, com ou sem homogeneidade de variâncias e com ou sem balanceamento.

\section{REFERÊNCIAS BIBLIOGRÁFICAS}

BERRY, D.A. Multiple comparisons, multiple tests, and data dredging: a Bayesian perspective: with discussion. In: BERNARDO, J.M.; DEGROOT, M.H.; LINDLEY, D.V.; SMITH, A.F.M. Bayesian statistics. Oxford: Oxford University, 1988. v.3, p.79-94.

BERRY, D.A.; HOCHBERG, Y. Bayesian perspectives on multiple comparisons. Journal of Statistical Planning and Inference, Amsterdam, v.82, p.215-227, 1999. 
BRATCHER, T.; HAMILTON, C. A Bayesian multiple comparison procedure for ranking the means of normally distributed data. Journal of Statistical Planning and Inference, Amsterdam, v.133, p.23-32, 2005.

CHO, J.S.; MASOOM, M.A.; BEGUM, M. Bayesian multiple comparisons with nonparametric Dirichlet process prioris for negative binomial populations. Pakinson Journal Statistical, v.22, n.2, p.89-98, 2006.

DUNCAN, D.B. A Bayesian approach to multiple comparisons. Technometrics, Washington, v.7, p.171222, 1965.

GOPALAN, R.; BERRY, D.A. Bayesian multiple comparisons using Dirichlet process priors. Journal American Statistical Association, Washington, v.93, n.443, p.1130-1139, 1998.

HOCHBERG, Y.; TAMHANE, A.C.T. Multiple comparison procedures. New York: J.Wiley, 1987.

HSU, J.C. Multiple comparisons: theory and methods. London: CRC, 1996.
MACHADO, A.A.; DEMÉTRIO, C.G.B.; FERREIRA, D.F.; SILVA, J.G.C. Estatística experimental: uma abordagem fundamentada no planejamento e no uso de recursos computacionais. In: REUNIÃO ANUAL DA REGIÃO BRASILEIRA DA SOCIEDADE

INTERNACIONAL DE BIOMETRIA, 50.; SIMPÓSIO DE ESTATÍSTICA APLICADA À EXPERIMENTAÇÃO AGRONÔMICA, 11., 2005, Londrina. Anais... Londrina: ISBN, 2005 .

R DEVELOPMENT CORE TEAM. R: a language and environment for statistical computing. Vienna: $R$ Foundation for Statistical Computing, 2005. Disponível em: http://Www.R-project.org' Acesso em: 10 jun. 2006.

SHAFFER, P.J. A semi-Bayesian study of Duncan's Bayesian multiple comparison procedure. Journal of Statistical Planning and Inference, Amsterdam, v.82, p.197-213, 1999.

WALLER, R.A.; DUNCAN, D.B. A Bayes rule for the symmetric multiple comparisons problem. Journal of the American Statistical Association, Washington, v.64, n.328, p.1484-1503, 1969. 